\title{
Effective brain state estimation during propofol-induced sedation using advanced EEG microstate spectral analysis
}

\author{
Yamin Li, Wen Shi, Zhian Liu, Jing Li, Qiang Wang, Xiangguo Yan, Zehong Cao, Member, \\ IEEE and Gang Wang*, Member, IEEE
}

\begin{abstract}
Brain states are patterns of neuronal synchrony, and the electroencephalogram (EEG) microstate provides a promising tool to characterize and analyze the synchronous neural firing. However, the topographical spectral information for each predominate microstate is still unclear during the switch of consciousness, such as sedation, and the practical usage of the EEG microstate is worth probing. Also, the mechanism behind the anesthetic-induced alternations of brain states remains poorly understood. In this study, an advanced EEG microstate spectral analysis was utilized using multivariate empirical mode decomposition in Hilbert-Huang transform. The practicability was further investigated in scalp EEG recordings during the propofol-induced transition of consciousness. The process of transition from the awake baseline to moderate sedation was accompanied by apparent increases in microstate (A, B, and F) energy, especially in the whole-brain delta band, frontal alpha band and beta band. In comparison to other effective EEG-based parameters that commonly used to measure anesthetic depth, using
\end{abstract}

Manuscript received January 10, 2020. This work was supported in part by the Natural Science Basic Research Program of Shaanxi under Program No. 2020JM-037; in part by the National Natural Science Foundation of China under Grants 31571000, 81201162 and 61471291; and in part by the Fundamental Research Funds for the Central Universities of China under Grant xjj2017122. (Yamin Li and Wen Shi are co-first authors.) (Corresponding author: Gang Wang.)

Y. Li, W. Shi, Z. Liu, X. Yan, and G. Wang are with the Key Laboratory of Biomedical Information Engineering of Ministry of Education, Institute of Biomedical Engineering, School of Life Science and Technology, Xi'an Jiaotong University, Xi'an 710049, China, and also with National Engineering Research Center for Healthcare Devices, Guangzhou 510500 , China, and also with the Key Laboratory of Neuro-informatics and Rehabilitation Engineering of Ministry of Civil Affairs, Xi'an 710049, China. Y. Li and W. Shi are also with School of Biomedical Engineering and Med-X Research Institute, Shanghai Jiao Tong University, Shanghai 200240, China, and W. Shi is also currently with Key Laboratory for Biomedical Engineering of Ministry of Education, Department of Biomedical Engineering, College of Biomedical Engineering \& Instrument Science, Zhejiang University, Hangzhou 310027, China. (e-mail: ggwang@xitu.edu.cn)

$\mathrm{J}$. Li is with the Department of Anesthesiology, Honghui Hospital, Xi'an Jiaotong University, Xi'an 710054, China, and also with the Key Laboratory of Biomedical Information Engineering of Ministry of Education, Institute of Biomedical Engineering, School of Life Science and Technology, Xi'an Jiaotong University, Xi'an 710049, China.

Q. Wang is with Department of Anesthesiology and Center for Brain Science, First Affiliated Hospital, Xi'an Jiaotong University, Xi'an 710061, China.

Z. Cao is with the Discipline of ICT, School of Technology, Environments and Design, University of Tasmania, Hobart, TAS, 7001, Australia. the selected spectral features reached better performance ( $80 \%$ sensitivity, $90 \%$ accuracy) to estimate the brain states during sedation. The changes in microstate energy also exhibited high correlations with individual behavioral data during sedation. In a nutshell, the EEG microstate spectral analysis is an effective method to estimate brain states during propofol-induced sedation, giving great insights into the underlying mechanism. The generated spectral features can be promising markers to dynamically assess the consciousness level.

Index Terms-Electroencephalogram, microstate spectral analysis, multivariate empirical mode decomposition, sedation, transition of consciousness

\section{INTRODUCTION}

A $\mathrm{S}$ a cornerstone of modern medicine, general anesthesia (GA), a drug-induced and reversible condition comprising specific behavioral and physiological traits, is crucial for safely performing surgical and nonsurgical procedures [1], [2]. Although several types of anesthetic drugs have extensively used in clinical practice, the underlying spatial and temporal electrophysiology is not that clear during the entire process, and the mechanism of brain modulation during the anesthetic-induced transition of consciousness is still under debate. Furthermore, it is highly useful and desirable to accurately monitor the depth of GA, which helps assess the alteration of brain states and minimize the intra-operative awareness in clinical care and surgery. Most available methods are based on non-invasive functional brain imaging tools, including electroencephalogram (EEG), near-infrared spectroscopy (NIRs), and functional magnetic resonance imaging (fMRI) [3]-[6]. As EEG non-invasively measures scalp electrical potentials generated by the cortical postsynaptic current, it is a highly practical and powerful measurement for dynamically tracking brain states under GA for its high temporal resolution. Although several analytical methods are currently proposed as potential markers of consciousness, such as complexity [7], entropy [8], brain connectivity [9], phase-amplitude coupling [2], global coherence analysis [10] and bispectral index (BIS) [11], it is still of importance to explore other novel and effective markers that can help accurately track brain dynamics and better the understanding of the brain states during the transitions of consciousness.

EEG microstates are quasi-stable short periods, during which the global topography is fixed. Nevertheless, the strength can 
be different, and the polarity is negligible [12]. As a direct measure of the transient brain state, the EEG microstates are capable of representing the summation of concurrent active sources in the brain and indexing different types of the mental process [13]-[16], leaving great prospect in tracking the level of consciousness during anesthesia. Using topographical clustering methods, such as modified k-means and topographic atomize and agglomerate hierarchical clustering (TAAHC), typical maps are taken as the so-called microstates. These maps have been widely used to study the residual consciousness in unresponsive patients [17] and other consciousness-related brain activities, including sleep [18], [19], drowsiness [20], and hypnosis [21]. However, most of these researches focused on microstate analysis in a broad frequency range $(1 \sim 40 \mathrm{~Hz})$. In the previous study, GA was usually associated with significant shifts and changes in EEG power [1], [2], including the increase in frontal alpha power, i.e., anteriorization, as well as the progressive emergence of low-frequency activity [1], [2], [22]-[24]. Hence, it is of great interest to analyze the frequency component of the microstates and probe relevant topographical spectral information during anesthesia.

Conventional time-frequency (T-F) spectral analysis that employs Fourier and wavelet transform usually fails to analyze EEG signals with short lengths, such as EEG microstates that quickly switch within 60-120 milliseconds [25] [26]. Many studies employed Hilbert transform in microstate analysis for its feasibility in analyzing short-length signals in order to conquer those intrinsic restrictions [20], [27]. However, methodological problems still exist due to the meaningless negative instantaneous frequency generated by Hilbert transform and lack of precision in the broad frequency band [26], [28]. Recently, we proposed a novel microstate-wise spectral analysis method to investigate spectral information of non-canonical microstate during propofol-induced sedation [29], in which a multivariate empirical mode decomposition (MEMD) was incorporated with Hilbert-Huang transform (HHT) [28], [30] to tackle the aforementioned problems. The microstate-wise marginal spectrum was then generated for spectral analysis. However, the topographical spectral information underlying different types of microstate across different levels of consciousness was not apparent, and we strived to resolve the questions in this work.

Here, the advanced microstate method was systematically put forward as EEG microstate spectral analysis, and a comprehensive investigation was performed on the topographical frequency patterns of different EEG microstate classes. The feasibility of the methodology was also validated by applying it to the brain state estimation during the propofol-induced transition of consciousness. The novel method, based on spectral analysis of microstates, could 1) effectively extract regional frequency components of microstate; 2) uncover and complement the neural mechanism of dynamic brain electrical activities according to the prior microstate source localization; 3) yield distinct microstate-based features that further increase the accuracy of estimating different brain states during propofol-induced sedation. This method was demonstrated highly useful and effective in brain state estimation during sedation and provided additional insights towards EEG microstate rationale.

\section{METHODS}

\section{A. Subject and data acquisition}

High-density EEG data used in this study are freely available at the University of Cambridge Data Repository (https://www.repository.cam.ac.uk/handle/1810/252736). It contained well-preprocessed scalp EEG data from 20 healthy participants (9 male, age: $30.85 \pm 10.98 \mathrm{yr}$ ), and the corresponding behavioral data were also provided.

The experiment began with an awake baseline (BS), and a computerized syringe driver (Alaris Asena PK, Carefusion, Berkshire, UK) was then utilized to control the infusion of propofol and maintain the target concentration. Three blood plasma levels were targeted at $0.6 \mu \mathrm{g} / \mathrm{ml}$ (mild sedation, ML), $1.2 \mu \mathrm{g} / \mathrm{ml}$ (moderate sedation, MD), and recovery (RC) from sedation. In each condition, approximately 7 minutes of 128-channel EEG data measured in microvolts were collected at a sampling rate of $250 \mathrm{~Hz}$ for each participant. Data from 91 channels over the scalp surface were retained for further analysis after the electrodes with severe movement-related noise were excluded. The data were then filtered between $0.5 \mathrm{~Hz}$ and $45 \mathrm{~Hz}$ and segmented into a series of 10 -second long epochs via EEGLAB [31]. Each epoch was baseline-corrected relative to the mean voltage over the entire epoch. Epochs containing excessive eye movement or muscular artifact were rejected by a quasi-automated procedure in EEGLAB [9], [31]. Finally, altogether a mean of 38, 39, 38, and 40 epochs were generated with a standard deviation of $5,4,4,2$ in the BS, ML, $\mathrm{MD}$, and $\mathrm{RC}$, respectively. During each stage of sedation, the participants were asked to perform a simple behavioral task to fast discriminate the type of auditory stimuli (buzz or noise) for forty times, and a correct response rate, as well as an average reaction time, were then calculated. Moreover, blood samples (1cc) each were taken at the beginning and end of mild and moderate sedation states, and once at recovery to obtain the actual propofol levels in plasma. Ethical approval for testing healthy controls was provided by the Cambridgeshire 2 Regional Ethics Committee. All clinical investigations were conducted following the Declaration of Helsinki. Other technical details are illustrated in Chennu's work [9].

\section{B. EEG microstate spectral analysis}

In this section, the EEG microstate spectral analysis is introduced. Firstly, EEG microstate sequences were calculated from high-density EEG signals. Secondly, MEMD and HHT were performed to estimate the instantaneous amplitude and frequency of original EEG signals, which could then be projected back to microstate sequences to obtain the segmented Hilbert spectrum. Finally, the microstate marginal spectrum and the baseline-normalized marginal mean energy topography were analyzed according to the Hilbert spectrum. The entire procedure of microstate spectral analysis is shown in Fig.1.

\section{1) Microstate analysis}

EEG microstate sequences were obtained following the standard microstate segmentation procedure. Firstly, the global field power (GFP) of EEG was calculated, and the EEG topographies at GFP peaks were then extracted because of the 


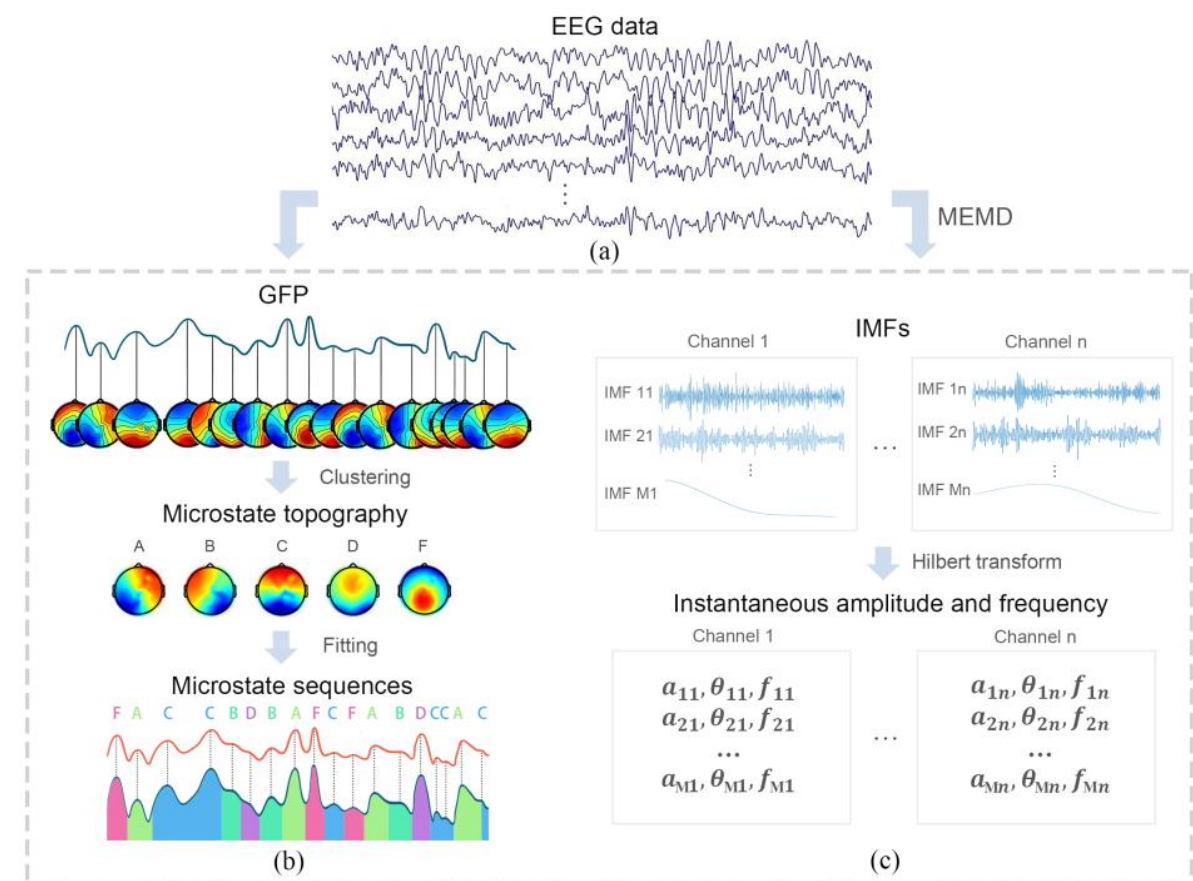

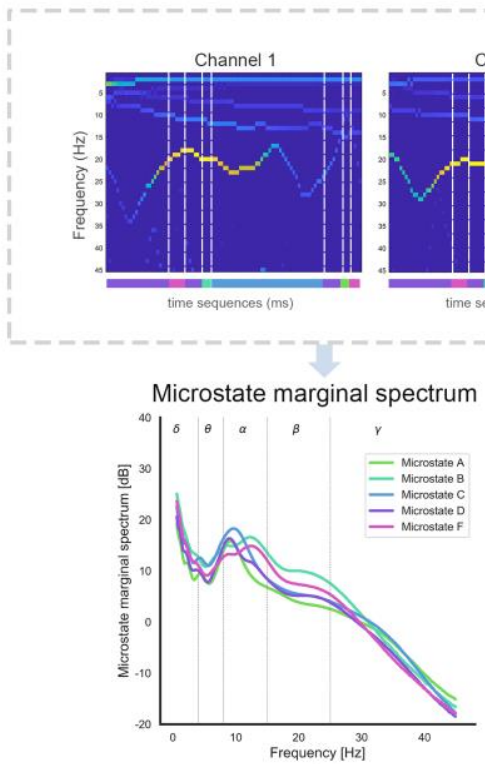

(e) (d)

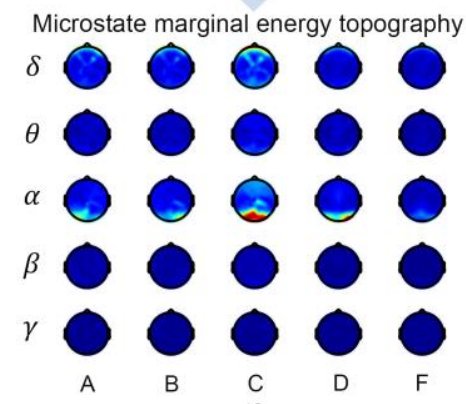

(f)

Fig. 1. Summary of microstate spectral analysis pipeline. (a) the original EEG signals. (b) The EEG microstate segmentation procedure (c) The calculation of instantaneous information of EEG. MEMD decomposed the EEG data into several IMFs, and instantaneous amplitude and frequency was subsequently obtained using the Hilbert transform. (d) EEG Hilbert spectrum with microstate sequences shown below. (e) The EEG microstate marginal spectrum. (f) The EEG microstate marginal energy topography.

highest signal to noise ratio. Templates within each epoch were computed using a spatial modified k-means clustering [25], [32]. Typical templates in all epochs were performed in a second clustering to obtain dominant microstates under each condition [12]. Subsequently, the spatial correlation was computed between microstates in baseline and those in other conditions, and the microstates in other stages were labeled as the name of the microstate with the highest spatial correlation in the baseline. Based on our previous study [29], five microstates were extracted using a modified k-means clustering algorithm, capturing more than $60 \%$ global explained variance, and were separately named as A, B, C, D, and F (see in Fig.2), according to the previous literature [13], [33]. Note that the average corresponding spatial correlation between baseline and other brain states is approximately $98 \%$. Finally, the original GFP peaks were labeled as one of these microstates according to the highest topographic correlation. [34]. A weak temporal smoothing was subsequently utilized in microstate sequences to ensure a certain degree of continuity. Then, each EEG time point was marked as the label of the nearest GFP peak. The first and the last GFP peaks of each epoch were excluded from fitting as their durations were uncertain [27], [35].

2) Microstate power

Microstate power of $N_{e}$-channel EEG signal is defined as 
below,

$$
\begin{gathered}
P_{m}=\frac{\sum_{t=1}^{L_{s}} \sum_{n=1}^{N_{e}}\left\|s_{n}(t)\right\|^{2} \cdot \gamma_{m, t}}{L_{s}}, \\
\gamma_{m, t}=\left\{\begin{array}{l}
1, \text { if time } \mathrm{t} \text { is labeled as microstate } \mathrm{m} \\
0, \text { otherwise }
\end{array}\right.
\end{gathered}
$$

where $P_{m}$ represents the power of microstate $m$, and $s_{n}(t)$ represents the EEG signals of electrode $n$. Additionally, $L_{s}$ is the total time of microstate sequences. The power of the signal equals the sum of power for all microstate classes. In this work, the microstate power was calculated in the given frequency band $(0.5 \sim 45 \mathrm{~Hz})$.

\section{3) Hilbert-Huang transform}

The conventional T-F methods are not necessarily appropriate to combine with microstate analysis. Real-world EEG is non-stationary signals, and the length of one microstate segment is relatively short [36]. Some processing steps even change the original topological patterns (e.g., filtering), which might lead to misinterpretation of the microstate topography. In this study, Hilbert-Huang transform (HHT) with multivariate empirical mode decomposition (MEMD) was performed to compute the instantaneous components of EEG signals in that it can obtain relatively meaningful instantaneous frequency for multi-channel EEG [28], [36]. Standard EMD decomposes data into a linear combination of intrinsic oscillatory modes, namely intrinsic mode function (IMF). However, for multi-channel data such as EEG, the local maxima and minima cannot be defined directly, and the notion of IMFs for multivariate signals remains ambiguous for EMD [37]. Thus, MEMD is employed to generate multiple $\mathrm{N}$-dimensional envelopes by taking signal projections along with different directions in $\mathrm{N}$-dimensional space, which are then averaged to obtain the local mean. For general simplicity, $N_{e}$-channel EEG signals at time $t$ can be designated as $\mathbf{s}(t)=\left[s_{1}(t), s_{2}(t), \ldots, s_{N_{e}}(t)\right] \quad, \quad$ and $\mathbf{v}^{\theta_{k}}=\left[v_{1}^{k}, v_{2}^{k}, \ldots, v_{N_{e}}^{k}\right]$ denotes a set of direction vectors along the direction given by angles $\boldsymbol{\theta}^{k}=\left[\theta_{1}^{k}, \theta_{2}^{k}, \ldots \theta_{\left(N_{e}-1\right)}^{k}\right]$ on a $\left(N_{e}-1\right)$ sphere. The main procedure is given below.

Firstly, generate a K-point for sampling on a $\left(N_{e}-1\right)$ sphere uniformly. Secondly, the $k$ th projection $p^{\theta_{k}}(t)$ of the input signal $\mathbf{s}(t)$ is calculated as $p^{\theta_{k}}(t)=\mathbf{s}(t) \cdot\left(\mathbf{v}^{\theta_{k}}\right)^{T}$, for all $k(k=$ $1,2, \ldots, K)$. Then find the time instants $\left\{t_{i}^{\theta_{k}}\right\}$ which correspond to the maxima of $p^{\theta_{k}}(t)$ for all $\mathrm{K}$ and multivariate envelope curves $\mathbf{e}^{\theta_{k}}(t)$ can be obtained by interpolating $\left[t_{i}^{\theta_{k}}, \mathbf{s}\left(t_{i}^{\theta_{k}}\right)\right]$. For a set of $\mathrm{K}$ direction vectors, the mean of envelope curves $\mathbf{m}(t)$ is calculated as below:

$$
\mathbf{m}(t)=\frac{1}{K} \sum_{k=1}^{K} \mathbf{e}^{\theta_{k}}(t) .
$$

Once the mean signal is defined, the rest of the procedure is quite similar to the standard EMD. Specifically, the "detail" $\boldsymbol{d}(t)$ is then extracted through $\boldsymbol{d}(t)=\mathbf{s}(t)-\boldsymbol{m}(t)$. If the "detail" $\boldsymbol{d}(t)$ fulfills the stopping criterion for a multivariate IMF [38], apply the above procedure to $\boldsymbol{x}(t)-\boldsymbol{d}(t)$, otherwise apply it to $\boldsymbol{d}(t)$. As a result, MEMD decomposes the $N_{e}$-channel EEG into several vector-valued forms of $\mathrm{IMFs} \mathbf{c}_{i}(t)$ with joint rotational modes and relevant residual $\mathbf{r}(t)$ by

$$
\mathbf{s}(t)=\sum_{i=1}^{M} \mathbf{c}_{i}(t)+\mathbf{r}(t), \mathbf{s}, \mathbf{c}_{i}, \mathbf{r} \in \mathbb{R}^{n},
$$

where the $N_{e}$-variate MIMFs, $\left\{\mathbf{c}_{i}(t)\right\}_{i=1}^{M}$, contain scale-aligned intrinsic joint rotational modes [26]. Hilbert transform is then applied to each IMF to obtain the instantaneous phase and frequency. The Hilbert transform on $n$th channel for $i$ th IMFs $c_{i n}(t)$ is given by

$$
H\left(c_{i \mathrm{n}}(t)\right)=\frac{1}{\pi} P \int_{-\infty}^{\infty} \frac{c_{i n}(\tau)}{t-\tau} d \tau,
$$

where $P$ denotes as Cauchy's principal value. Then, the amplitude $a_{i n}(t)$, phase $\theta_{i n}(t)$, and frequency functions $\omega_{i n}(t)$ can be easily obtained from the following equations for subsequent microstate spectral analysis:

$$
\begin{gathered}
a_{i n}(t)=\left|c_{i n}(t)+j H\left(c_{i n}(t)\right)\right|, \\
\theta_{i n}(t)=\arctan \left(\frac{H\left(c_{i n}(t)\right)}{c_{i n}(t)}\right), \\
\omega_{i n}(t)=\frac{d \theta_{i n}(t)}{d t} .
\end{gathered}
$$

Then, the $n$th channel signal of original EEG can be expressed as below, where $\operatorname{Re}\{$.$\} represents the real part of a complex$ quantity:

$$
s_{n}(t)=\operatorname{Re}\left\{\sum_{i=1}^{k} a_{i n}(t) \mathrm{e}^{j \int \omega_{i n}(t) d t}\right\}
$$

Then, the Hilbert spectrum $H_{n}(\omega, t)$ for $n$th channel of EEG can be computed according to (8). The Hilbert energy spectrum can be thus calculated in which the amplitude is substituted by its square value [30].

\section{4) Microstate marginal spectrum}

In this study, the Hilbert energy spectrum was acquired by the above standard HHT procedure and then partitioned into several segments according to microstate-labeled time sequences. The Hilbert energy spectrum from the same microstate class was added up and divided by the total length of microstate sequence. In the calculation of the marginal spectrum, the location of electrodes was ignored, and the energy of all the channels was summated. In sum, the Hilbert energy spectrum for a given microstate $m$ (microstate marginal spectrum) in the timeframe of interest was calculated by the equation below:

$$
h_{m}(\omega)=\frac{\sum_{n=1}^{N_{e}} \int_{T_{m}} H_{n}(\omega, t)^{2} d t}{L_{s}},
$$

where $T_{m}$ represents the timeframe that labeled as microstate $m$ and $L_{s}$ is the total length of the analyzed microstate sequences. In this study, the microstate marginal spectrum was calculated for each participant in each condition at certain frequency scales. The energy spectrum was divided into five frequency bands, namely the delta $(0.5-4 \mathrm{~Hz})$, theta $(4-8 \mathrm{~Hz})$, alpha $(8-15 \mathrm{~Hz})$, beta $(15-25 \mathrm{~Hz})$, and gamma $(25-45 \mathrm{~Hz})$ bands. 
Subsequently, the marginal energy for microstate $\mathrm{m}$ within a given range of frequency band ( $\Delta \omega$ ) could be obtained by the following equation:

$$
E_{m}=\int_{\Delta \omega} h_{m}(\omega) d \omega .
$$

\section{5) Microstate marginal energy topography}

As for the calculation of microstate marginal energy topography, the spatial information of electrodes was retained, and other computations were the same as the microstate marginal spectrum. The Hilbert energy spectrum for microstate $m$ at the $n$th channel was calculated as below:

$$
h_{m n}(\omega)=\frac{\int_{T_{m}} H_{n}(\omega, t)^{2} d t}{L_{s}},
$$

The microstate marginal energy topographies were calculated individually. Then, the baseline-normalization was performed by the channel-wise division between EEG marginal energy in other conditions and that in baseline. The topographies from all participants were averaged at each condition.

\section{C.Brain state estimation}

As a mature anesthetic marker, EEG-derived bispectral index (BIS) is widely utilized to track the levels of GA in clinical surgery [11]. It is computed from several important EEG parameters, including relative beta ratio (BetaRatio) and relative synchrony of fast and slow wave (SynchFastSlow) [39], [40], which are usually calculated from the EEG signal in the Fp1 channel. BetaRatio is defined as the logarithm ratio of the EEG spectral power within 30 to $47 \mathrm{~Hz}$ to the EEG spectral power in the 11 to $20 \mathrm{~Hz}$. SynchFastSlow is the logarithm ratio of the bispectral power in the frequency band $40-47 \mathrm{~Hz}$ to that in the band $0.5-47 \mathrm{~Hz}$. In this study, a simple linear-kernel support vector machine (SVM) with penalty parameter $\mathrm{C}=10$ was performed to classify the brain states, i.e., $\mathrm{BS}$ and $\mathrm{MD}$, in order to assess the potential of the microstate marginal energy in estimating the brain states during sedation. The results were also compared to other traditional markers, i.e., BetaRatio, SynchFastSlow. Note that all the features were baseline-normalized to reduce individual variability. To make fair comparisons with the classification using only BetaRatio or SynchFastSlow, we solely took marginal energy of single

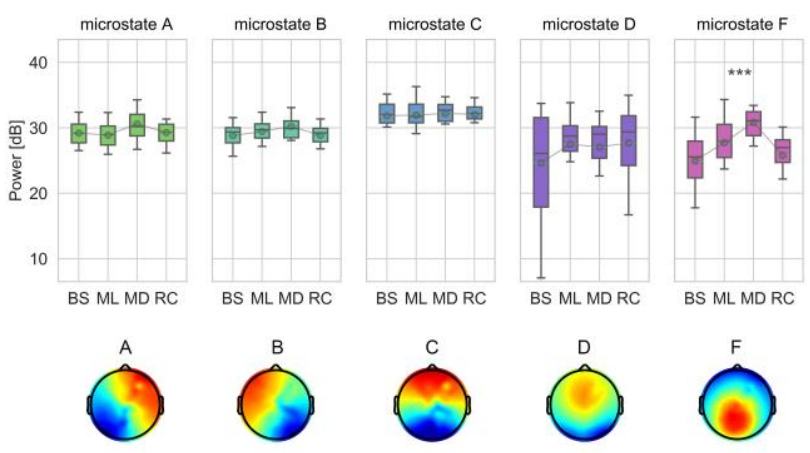

Fig. 2. Microstate topographies in the baseline and the boxplots of corresponding power fluctuation. The average power is linked by solid grey lines across conditions. $\mathrm{BS}=$ baseline, $\mathrm{ML}=$ mild sedation, $\mathrm{MD}=$ moderate sedation, $\mathrm{RC}=$ recovery. ${ }^{* * *}: \mathrm{p}<0.001$

microstate class in one frequency band as the only feature in each classification. Leave-one-out cross-validation (LOOCV) was used to validate the performance of classifications. Each loop in LOOCV generated a testing result. The relevant metrics (sensitivity, specificity, accuracy, and F1-score) of the testing results were reported.

\section{D.Statistic}

In this study, Matlab 2019b (MathWorks, Natick, MA) Statistics Toolbox was utilized to perform the statistical analysis. P values less than 0.05 were reported as significant. Repeated measures ANOVAs were implemented to see whether conditions (targeted plasma concentration) influenced microstate energy. The greenhouse-Geisser correction was used for correcting $p$ values if Mauchly's test of sphericity was violated. Kruskal-Wallis test was employed to examine the univariate effect of microstate on marginal energy within a given condition and frequency band. Linear regression was applied to explore the relationship between behavioral parameters and band energy in moderate sedation.

\section{RESULtS}

\section{A. EEG microstate marginal spectrum}

The microstate topographies in the baseline and the corresponding power in each condition are shown in Fig.2. The
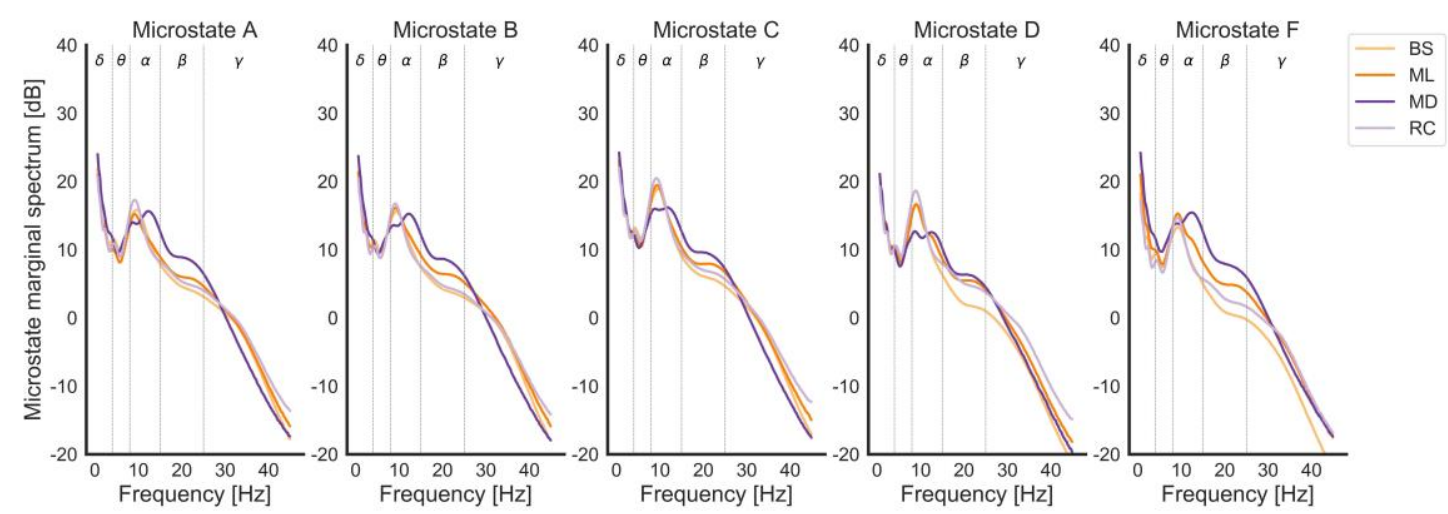

Fig. 3. The microstate marginal spectrum for each microstate and condition. The marginal spectrums from $0.5-45 \mathrm{~Hz}$ are plotted, with four conditions depicted in different colors. The solid lines represent the mean microstate marginal energy across all participants. $\mathrm{BS}=$ baseline, $\mathrm{ML}=$ mild sedation, $\mathrm{MD}=$ moderate sedation, $\mathrm{RC}=$ recovery. 
TABLE I

UNIVARIATE EFFECT OF MICROSTATE ON MARGINAL ENERGY WITHIN EACH GIVEN CONDITION AND FREQUENCY BAND

\begin{tabular}{llllll}
\hline \hline Condition & delta & theta & alpha & beta & gamma \\
\hline BS & $29.297(<\mathbf{0 . 0 0 1})$ & $17.962(\mathbf{0 . 0 0 1})$ & $19.181(<\mathbf{0 . 0 0 1})$ & $18.002(\mathbf{0 . 0 0 1})$ & $23.064(<\mathbf{0 . 0 0 1})$ \\
ML & $15.856(\mathbf{0 . 0 0 3})$ & $12.480(\mathbf{0 . 0 1 4})$ & $10.862(\mathbf{0 . 0 2 8})$ & $6.531(0.163)$ & $9.629(\mathbf{0 . 0 4 7})$ \\
MD & $8.954(0.062)$ & $9.061(0.060)$ & $11.638(\mathbf{0 . 0 2 0})$ & $6.739(0.150)$ & $5.075(0.280)$ \\
RC & $31.759(<\mathbf{0 . 0 0 1})$ & $19.914(<\mathbf{0 . 0 0 1})$ & $23.661(<\mathbf{0 . 0 0 1})$ & $13.130(\mathbf{0 . 0 1 1})$ & $14.470(\mathbf{0 . 0 0 6})$ \\
\hline \hline
\end{tabular}

Chi square ( $p$ value) is shown in table. $P$ values under 0.05 are in bold.

$\mathrm{BS}=$ baseline, $\mathrm{ML}=$ mild sedation, $\mathrm{MD}=$ moderate sedation, $\mathrm{RC}=$ recovery .

power of microstates $\mathrm{A}$ and $\mathrm{B}$ showed a growth trend (A: $\mathrm{p}=0.051 ; \mathrm{B}: \mathrm{p}=0.071)$ during sedation, while the power of microstate $F$ presented a significant enhancement $(p<0.001)$ when the targeted propofol plasma level increased. The changes reversed in recovery. Fig. 3 illustrates the EEG microstate marginal spectrum across conditions acquired by MEMD-embedded HHT. Overall, apparent energy reduction in the low alpha band $(8-12 \mathrm{~Hz})$, especially in microstates $\mathrm{C}$ and $\mathrm{D}$, and an increase in the beta band can be observed in moderate sedation. For microstates A, B, and F, moderate sedation was also accompanied by notable energy enhancement in the delta and high alpha band $(12-15 \mathrm{~Hz})$. The energy increase for microstate F, particularly in the delta, alpha, and beta bands, was the most remarkable.

To precisely and quantitatively characterize the microstate energy distribution in certain frequency bands during the transition of consciousness, the marginal spectrum was added up according to the range of these frequency bands. Interestingly, the sedation had significantly influenced on the energy of microstate $A$ in the delta $(p=0.043)$ and the beta $(p=0.009)$ bands, the energy of microstates $B(p=0.009)$ and $C$ $(p=0.004)$ in the beta band, the energy of microstate $D$ in the beta $(\mathrm{p}=0.020)$ and the gamma band $(\mathrm{p}=0.047)$, and energy of microstate $\mathrm{F}$ for all bands (delta: $\mathrm{p}=0.001$; theta: $\mathrm{p}=0.025$; alpha: $\mathrm{p}=0.002$; beta: $\mathrm{p}<0.001$; gamma: $\mathrm{p}<0.001$ ). These significant changes in microstate energy all displayed an enhancement as sedation progressed, which reversed in recovery. Also, Table I illustrates the quantitative comparison of marginal energy across different microstates within each given condition and frequency band. Kruskal-Wallis tests indicate that, in the baseline and recovery, energy in all frequency bands was significantly different across microstates, while in mild sedation, the phenomenon disappeared in the beta band. In moderate sedation, only the alpha marginal energy was significantly different across these microstates.

\section{B.EEG microstate marginal energy topography}

In order to better characterize location-specific energy distribution and fluctuation, the baseline-normalized microstate energy topographies for each frequency band during sedation were calculated (see Fig.4). First, for microstates A and B, significant energy fluctuation mainly took place in the delta, alpha, and beta band. Concretely, the alpha energy increased in the frontal area, while beta energy enhanced globally with higher frontal amplification. As for microstate C, only beta energy significantly enhanced in the frontal area during

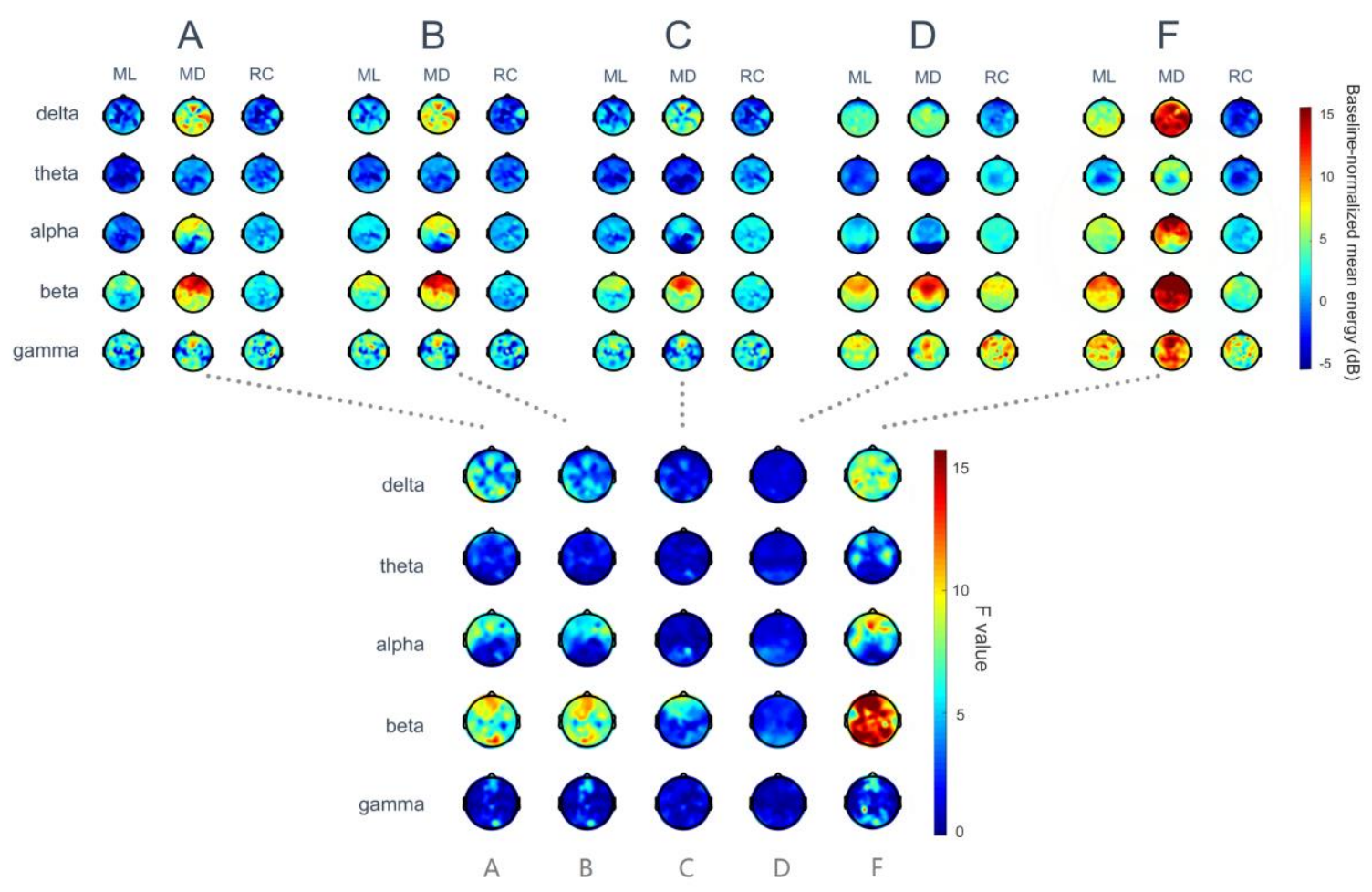

Fig. 4. Baseline-normalized mean marginal energy topography of each microstate on five frequency bands ( $F$ value maps from repeated measures ANOVAs are shown). $\mathrm{BS}=$ baseline, $\mathrm{ML}=$ mild sedation, $\mathrm{MD}=$ moderate sedation, $\mathrm{RC}=$ recovery. 
TABLE II

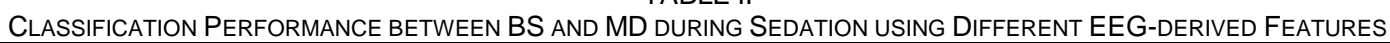

\begin{tabular}{lllllllll}
\hline \hline Method & TP & TN & FP & FN & Sensitivity & Specificity & Accuracy & F1-score \\
\hline SynchFastSlow & 12 & 20 & 0 & 8 & 0.60 & $\mathbf{1 . 0 0}$ & 0.80 & 0.75 \\
BetaRatio & 14 & 20 & 0 & 6 & 0.70 & $\mathbf{1 . 0 0}$ & 0.85 & 0.82 \\
microstate A-beta & 14 & 20 & 0 & 6 & 0.70 & $\mathbf{1 . 0 0}$ & 0.85 & 0.82 \\
microstate A-gamma & 13 & 20 & 0 & 7 & 0.65 & $\mathbf{1 . 0 0}$ & 0.83 & 0.79 \\
microstate B-beta & 15 & 20 & 0 & 5 & 0.75 & $\mathbf{1 . 0 0}$ & 0.88 & 0.86 \\
microstate F-beta & 16 & 20 & 0 & 4 & $\mathbf{0 . 8 0}$ & $\mathbf{1 . 0 0}$ & $\mathbf{0 . 9 0}$ & $\mathbf{0 . 8 9}$ \\
\hline \hline
\end{tabular}

SycnFastSlow = relative synchrony of fast and slow wave, $\mathrm{BS}=$ baseline, $\mathrm{MD}=$ moderate sedation.

$\mathrm{TP}=$ true positive, $\mathrm{TN}=$ true negative, $\mathrm{FP}=$ false positive, $\mathrm{FN}=$ false negative.

TABLE III

The Pearson CorRelation betWeen BehaVioral Data AND Microstate Marginal EneRgy In THE Moderate Sedation

\begin{tabular}{llrrr}
\hline \hline \multirow{2}{*}{ Band } & MS & Responsive rate & Reaction time & Drug in blood \\
& & & & \\
\hline$\delta$ & $\mathrm{A}$ & $-0.588 \mathbf{( 0 . 0 0 6})$ & $0.359(0.120)$ & $0.567(\mathbf{0 . 0 0 9})$ \\
& $\mathrm{B}$ & $-0.539(\mathbf{0 . 0 1 4})$ & $0.346(0.134)$ & $0.585(\mathbf{0 . 0 0 7})$ \\
$\theta$ & $\mathrm{F}$ & $-0.677(\mathbf{0 . 0 0 1})$ & $0.210(0.374)$ & $0.485(\mathbf{0 . 0 3 0})$ \\
& $\mathrm{A}$ & $-0.288(0.219)$ & $0.563(\mathbf{0 . 0 1 0})$ & $0.313(0.178)$ \\
& $\mathrm{B}$ & $-0.173(0.464)$ & $0.560(\mathbf{0 . 0 1 0})$ & $0.321(0.168)$ \\
$\alpha$ & $\mathrm{F}$ & $-0.349(0.131)$ & $0.564 \mathbf{( 0 . 0 1 0})$ & $0.210(0.372)$ \\
& $\mathrm{A}$ & $-0.315(0.177)$ & $0.623 \mathbf{( 0 . 0 0 3})$ & $0.523(\mathbf{0 . 0 1 8})$ \\
$\beta$ & $\mathrm{B}$ & $-0.251(0.284)$ & $0.657(\mathbf{0 . 0 0 2})$ & $0.489(\mathbf{0 . 0 2 9})$ \\
$\beta$ & $\mathrm{F}$ & $-0.293(0.209)$ & $0.692(\mathbf{0 . 0 0 1})$ & $0.434(0.056)$ \\
& $\mathrm{A}$ & $0.000(0.969)$ & $0.383(0.096)$ & $0.290(0.217)$ \\
$\gamma$ & $\mathrm{B}$ & $-0.032(0.900)$ & $0.431(0.058)$ & $0.327(0.160)$ \\
& $\mathrm{F}$ & $-0.045(0.864)$ & $0.505(\mathbf{0 . 0 2 3})$ & $0.292(0.213)$ \\
& $\mathrm{A}$ & $0.389(0.090)$ & $0.170(0.475)$ & $0.000(0.955)$ \\
& $\mathrm{B}$ & $0.373(0.106)$ & $0.158(0.508)$ & $0.000(0.993)$ \\
\hline \hline
\end{tabular}

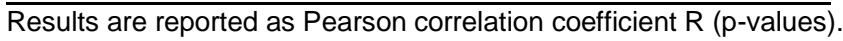

$P$ values under 0.05 are in bold. MS = Microstate

sedation. Lastly, mean energy for microstate $\mathrm{F}$ fluctuated the most obviously during consciousness alternation in different regions. The marginal energy of microstate $\mathrm{F}$ in the delta and beta band significantly enhanced in the global region, with a remarkable increase of alpha band energy in the frontal area.

\section{C.State assessment and behavior correlations}

Table II shows the statistics of the classification between the two brain states (BS and MD) during sedation using different EEG-derived features. Note that only the microstate spectral features with better classification accuracy than SynchFastSlow were listed. It reflected that marginal energy for microstate $\mathrm{F}$ in the beta band achieved the highest sensitivity $(0.80)$, accuracy $(0.90)$, and F1-score (0.89) than any other feature. In addition, many microstate spectral features outperformed the conventional EEG-derived parameters (SynchFastSlow and BetaRatio) that monitored the level of consciousness during sedation.

Pearson correlations between microstate marginal energy in the specific band and behavioral parameters in moderate sedation are illustrated in Table III. Note that only the results of microstates $\mathrm{A}, \mathrm{B}$, and $\mathrm{F}$, whose marginal energy significantly correlated with behavioral data, are shown. Participants who were difficult to discriminate the acoustic stimuli accurately had high delta energy of microstates A, B, and F, accompanied by low $\mathrm{F}$ gamma energy conversely. Likewise, the longer reaction time to the stimuli, the higher theta and alpha energy for microstates A, B, and F, and beta energy for microstate F.
The microstate energy was also associated with the physical targets, such as propofol concentration in blood. Specifically, a high positive correlation was found between plasma drug concentration and delta, alpha energy for microstate A, B, and F.

\section{DISCUSSION}

It is assumed that microstate topographies are determined by the brain activities generated from specific oscillation sources in the inter-cortical regions. However, their spectral information is still lack of investigation, especially during transitions of brain states [41]. In this study, the findings implied the association between cerebral functional dynamics and the spectral information, driven by EEG microstate marginal spectrum and mean energy topography during the propofol-induced transition of consciousness. The results revealed great insights into the underlying mechanism, offered promising markers, and provided effective measurement to estimate the brain states during sedation.

\section{A. Microstate marginal spectral analysis}

EEG microstate power can directly reveal the power alternation across conditions in a broad frequency band $(0.5 \sim 45 \mathrm{~Hz})$ for each microstate class. The power of the microstate $\mathrm{A}$ and $\mathrm{B}$ tended to rise, and the power of $\mathrm{F}$ significantly increased $(p<0.001)$ when the sedation progressed. Nonetheless, the power of $\mathrm{C}$ and $\mathrm{D}$ remained relatively stable. These implied that the total power of EEG signals increased during sedation [24] as the microstate state power was additive. The microstate marginal spectrum further reflected the energy fluctuations across frequency for specific microstate patterns and conditions. The enhanced beta band energy during moderate sedation was discovered in all microstates (see Fig. 3). Besides, the minimum marginal energy difference between microstates was found in the beta band compared with other frequency bands (see Table I). Hence, the elevated beta energy during propofol-induced sedation might be associated with all the microstates. It also implied that beta oscillation in the brain might not be generated by certain regions but could be a general feature of neural electrical activities. A similar phenomenon was found in Milz's research that there were very limited voxels significantly different in the beta band that primarily associated with facilitation [27]. Additionally, Milz et al. also demonstrated that the intra-cortical alpha oscillations primarily accounted for the most differences between microstate classes [27]. Similarly, in this work, alpha energy was the only pattern that could significantly differentiate the five microstates (as 
TABLE IV

SUMMARY OF MICROSTATE MARGINAL ENERGY FEATURES DURING SEDATION

\begin{tabular}{ll}
\hline $\begin{array}{l}\text { Mean marginal } \\
\text { energy }\end{array}$ & Neural phenomena from BS to MD \\
\hline A - delta & Energy exhibited an increasing trend globally. \\
\hline F - delta & $\begin{array}{l}\text { A significant elevation of energy broadly } \\
\text { distributed across the entire scalp. }\end{array}$ \\
\hline A - alpha & $\begin{array}{l}\text { Slight energy increase in the frontal part, } \\
\text { without significant change in marginal } \\
\text { energy. }\end{array}$ \\
\hline B - alpha & $\begin{array}{l}\text { Energy enhanced significantly, especially in } \\
\text { the frontal area. }\end{array}$ \\
\hline F - alpha - beta & $\begin{array}{l}\text { Energy enhanced globally, and significantly } \\
\text { in the frontal region. }\end{array}$ \\
B - beta & $\begin{array}{l}\text { Energy enhanced slightly in the frontal area. } \\
\text { C - beta }\end{array}$ \\
\hline F - beta & $\begin{array}{l}\text { Significant energy enhancement took place } \\
\text { across the scalp, especially in the frontal } \\
\text { region. }\end{array}$ \\
\hline \hline
\end{tabular}

All phenomena reversed in the recovery.

$\mathrm{BS}=$ baseline, $\mathrm{MD}=$ moderate sedation .

shown in Table I) in moderate sedation, corroborating Milz's findings from a different angle. Interestingly, the significant difference of marginal energy across microstates in five frequency bands was narrowed in the sedation. Further researches are needed to unravel the underlying mechanism of this phenomenon.

As the microstate marginal spectrum added up the energy of all channels, spatial information of microstate energy was eliminated. Thus, the microstate marginal energy topography was utilized to reflect the location-specific energy distribution and fluctuation of each microstate. Similar to the results of the spectrum in the beta band, a significant whole-brain energy increase was found in all microstates. Moreover, mean energy in the delta and alpha band also enhanced for microstates A, B, and F. Particularly, enhancements in the alpha and beta bands lay in the frontal area (see Fig.4). In the previous findings, topographical features in the alpha, beta, and gamma bands were vital in tracking brain states during propofol-induced sedation [2]. Using spectrograms, researchers have found that sedation and loss of consciousness (LOC) in propofol-induced anesthesia were usually parallel with increasing alpha, beta and gamma mean energy, particularly in the anterior region of the brain, which is also considered to be a manifestation of anteriorization [2], [10], [22]-[24], [42]. Specifically, it was observed that EEG mean energy would significantly increase in the frontal regions of the brain in the gamma band, and then shift to the beta band before participants lost their consciousness. Once the brain state transitioned to unconsciousness, this frontal energy pattern traveled to the beta and then alpha bands along with a decreasing trend in the median frequency (the gamma band). Note that in this study, not all participants lost consciousness because some of them still responded to the stimuli in the moderate sedation. Thus, it was reasonable that the alpha and beta energy increased in microstate energy topography. Second, the elevated slow-delta oscillation is a well-known marker of propofol-induced sedation, reflecting a state with reduced cortical-cortical connectivity [2], [43]-[45]. Generated by specific cortical and thalamic neurons, these slow oscillations occurred asynchronously across the cortex, creating a state of functional isolation between cortical areas and hindering cortical communication [42], [44]. This typical pattern was also found mainly for microstate $\mathrm{F}$ in moderate sedation, also indicating the unique role the microstate $\mathrm{F}$ played during the transition of consciousness [29]. Additionally, we didn't find significant decreases in microstate marginal energy in any of the frequency bands during sedation (see Table IV).

Former studies combined fMRI, EEG source imaging, as well as EEG microstate analysis to explore the relationship between functional brain network and certain microstate topography [33], [41]. In this work, microstate F had the most significant mean marginal energy alteration during sedation, which we have already interpreted in our previous work [29]. The results revealed that the increased energy of delta oscillation and the anteriorization in the alpha band, which were widely found in propofol-induced sedation and anesthesia [2], [42], possibly resulted from the intercortical activity of microstate F (see Fig.4). The significant enhancement in energy for microstate F was likely driven by the ACC cortical activity because intense activation in the dorsal anterior cingulate cortex (ACC) was associated with microstate F, according to the microstate source localization based on a large population [33]. The dorsal ACC was demonstrated as a part of frontoparietal networks, where the corticocortical and thalamocortical connectivity significantly decreased together with the decrease in consciousness during propofol-induced sedation [46]. The thalamus-related activity, especially the altering connectivity between the thalamus and ACC, is crucial in sedation modulation [23], [47], [48]. Therefore, the enhanced mean marginal energy for $\mathrm{F}$ might be owed to dysfunctional modulations in thalamocortical and corticocortical interaction during sedation and LOC [44], [46], [49]. In addition to microstate $\mathrm{F}$, the mean marginal energy of microstates A and B also enhanced, especially in the delta, alpha and beta bands. Though microstates A was demonstrated mainly linked with the primary auditory network in the temporal cortex, and B with the primary visual network in the occipital cortex [41], [50], the accurate regions contributing to microstate $A$ and $B$ are still unclear. In recent years, researchers found that the primary visual and auditory networks preserved and still responded to the stimuli, rather than dissociated when sedation progressed [46]. However, the ability for higher-level analysis for these sensory signals was lost, which might attribute to the potentiated $\gamma$-aminobutyric acid-mediated inhibition at multiple levels of the ascending sensory pathways, including the thalamus and cortex [51]. Similarly, in our results, most of the enhancement of A and B were not in these primary sensory cortices. Thus, we speculate that a higher-order network, in addition to the primary visual and auditory regions, might also be involved in the formation of microstates A and B. In other words, microstates A and B could reflect a higher-level activity or relatively global network rather than only mirroring the activities in early sensory cortices. These high-order networks altered during sedation, which might contribute to the rising trend of mean marginal energy of microstates A and B. Since there is very limited literature about the cortices and network corresponding to each microstate, and the sources of microstate are still under exploration, the underlying 
mechanism is still an open question. Also, only a small number of studies have reported and discussed the non-canonical microstate $F$ in the resting state [29], [33]. Thus, further researches combining multi-modality are still required to investigate and validate the mechanism and localization.

\section{B. Potential of quantitative assessing brain states}

Accurately tracking brain states is significant during GA in contemporary clinical medicine. In this study, the microstate marginal energy altered as the sedation progressed, indicating that these phenomena can potentially track the level of consciousness. The relevant features are summarized in Table $\mathrm{IV}$, and we then compared the classification capability based on the features and another two well-acknowledged brain state indicators, namely SynchFastSlow and BetaRatio, during sedation. The traditional markers are highly associated with the BIS index, a precise and widely used measurement of brain states during GA. The classification results showed that the microstate-based spectral features, especially the marginal energy of microstate $\mathrm{F}$ in the beta band, achieved better performance than either SynchFastSlow or BetaRatio. It had great potential in differentiating brain states during the transition of consciousness (see Table II), and could serve as the prospective marker that translated into clinical surgery, though further validation is required. Besides, we noticed that these well-behaved microstate features generated from the novel method mainly came from the beta band and gamma band, and BetaRatio was also calculated from the two bands. It further demonstrated that the marginal energy in the beta and gamma band was useful anesthetic signatures, and microstate-wise spectral analysis could further enhance monitoring brain states.

The three types of behavioral data in this study, i.e., the accuracy of perceiving the stimuli, the reaction time for the stimuli, and the drug concentration, all reflected the consciousness level during sedation. Particularly, the reduction of accuracy for perceiving the stimuli was considered simultaneously paralleled by LOC, which was used to explore EEG spectral signatures of loss and recovery of consciousness from propofol [2]. As the marginal band energy of microstates $\mathrm{A}, \mathrm{B}$, and F, especially in the delta band, was highly associated with responsive hit rate in moderate sedation, the specific marginal energy could estimate consciousness level. Moreover, the individual responsiveness measured by reaction time could be predicted by microstate energy, especially in the alpha band, which offers an extra reference to get rid of awareness in anesthetic-induced surgery. Likewise, increased blood levels of drug concentration was also correlated to the enhanced delta and alpha energy for microstates A, B, and F. Due to individual variability in susceptibility to anesthetic dosage, EEG can probably serve as an effective indicator to measure the effect of propofol on the brain and tailor optimal anesthetic dose for individuals during GA. Using accelerated MEMD [52], all these features have great potential to become individualized for dynamically assessing brain state and predicting the level of consciousness in clinical care. Further investigations are aimed to confirm their feasibility.

\section{V.CONCLUSION}

In this work, the global and local frequency components were extracted from each microstate class during propofol-induced sedation using the advanced microstate spectral analysis. The method could effectively estimate different brain states, and some intriguing results were spotted in EEG spatio-temporal dynamics. The transition of consciousness was accompanied by apparent energy increases of microstates A, B, and F, especially in the delta band, and frontal alpha and beta bands. The microstate-derived spectral features, such as the marginal energy of microstate $F$ in the beta band, have great capability in brain state estimation during sedation. The EEG microstate spectral analysis sheds new light to study the spatial and temporal spectral patterns of EEG microstates. It also generates promising markers that can assess the consciousness level, offering potential opportunities for applications in clinical medicine.

\section{REFERENCES}

[1] N. D. Brown, Emery N, and Ralph, Lydic and Schiff, "General anesthesia, sleep, and coma," N. Engl. J. Med., vol. 363, no. 27, pp. $2638-2650,2011$.

[2] P. L. Purdon et al., "Electroencephalogram signatures of loss and recovery of consciousness from propofol," Proc. Natl. Acad. Sci., vol. 110, no. 12, pp. E1142-E1151, 2013.

[3] I. J. Rampil, "A primer for EEG signal processing in anesthesia," Anesthesiology, vol. 89, no. 4, pp. 980-1002, 1998.

[4] G. Wang et al., "Monitoring the Depth of Anesthesia Through the Use of Cerebral Hemodynamic Measurements Based on Sample Entropy Algorithm," IEEE Trans. Biomed. Eng., vol. 67, no. 3, pp. 807-816, 2020.

[5] F. Cavanna, M. G. Vilas, M. Palmucci, and E. Tagliazucchi, "Dynamic functional connectivity and brain metastability during altered states of consciousness," Neuroimage, vol. 180, no. Pt B, pp. 383-395, 2018.

[6] P. Barttfeld, L. Uhrig, J. D. Sitt, M. Sigman, B. Jarraya, and S. Dehaene, "Signature of consciousness in the dynamics of resting-state brain activity," Proc. Natl. Acad. Sci., vol. 112, no. 3, pp. 887-892, 2015.

[7] X.-S. Zhang, R. J. Roy, and E. W. Jensen, "EEG complexity as a measure of depth of anesthesia for patients," IEEE Trans. Biomed. Eng., vol. 48, no. 12, pp. 1424-1433, 2001.

[8] J. Bruhn, H. Röpcke, and A. Hoeft, "Approximate entropy as an electroencephalographic measure of anesthetic drug effect during desflurane anesthesia," Anesthesiol. J. Am. Soc. Anesthesiol., vol. 92, no. 3, pp. 715-726, 2000.

[9] S. Chennu, S. O’Connor, R. Adapa, D. K. Menon, and T. A. Bekinschtein, "Brain Connectivity Dissociates Responsiveness from Drug Exposure during Propofol-Induced Transitions of Consciousness," PLoS Comput. Biol., vol. 12, no. 1, pp. 1-17, 2016.

[10] A. Cimenser et al., "Tracking Brain States under General Anesthesia by Using Global Coherence Analysis," Proc. Natl. Acad. Sci., vol. 108, no. 21, pp. 8832-8837, 2011.

[11] A. C. Searleman et al., "Anesthesia Awareness and the Bispectral Index," N. Engl. J. Med., vol. 358, no. 11, pp. 1097-1108, 2008.

[12] D. Van De Ville, J. Britz, and C. M. Michel, "EEG microstate sequences in healthy humans at rest reveal scale-free dynamics," Proc. Natl. Acad. Sci., vol. 107, no. 42, pp. 18179-18184, 2010.

[13] T. Koenig et al., "Millisecond by millisecond, year by year: Normative EEG microstates and developmental stages," Neuroimage, vol. 16 , no. 1, pp. 41-48, 2002.

[14] D. Lehmann and W. Skrandies, "Reference-free identification of components of checkerboard-evoked multichannel potential fields," Electroencephalogr. Clin. Neurophysiol., vol. 48, no. 6, pp. 609-621, 1980.

[15] D. Lehmann and W. Skrandies, "Spatial analysis of evoked potentials in man--a review," Prog. Neurobiol., vol. 23, no. 3, pp. 227-250, 1984. 
[16] J. Wackermann, D. Lehmann, C. M. Michel, and W. K. Strik, "Adaptive segmentation of spontaneous EEG map series into spatially defined microstates," Int. J. Psychophysiol. Off. J. Int. Organ. Psychophysiol., vol. 14, no. 3, p. 269, 1993.

[17] P. Gui et al., "Assessing the depth of language processing in patients with disorders of consciousness," Nat. Neurosci., vol. 23, no. 6, pp. 761-770, 2020.

[18] V. Brodbeck et al., "EEG microstates of wakefulness and NREM sleep," Neuroimage, vol. 62, no. 3, pp. 2129-2139, 2012.

[19] A. Kuhn, V. Brodbeck, and E. Tagliazucchi, "Narcoleptic Patients Show Fragmented EEG-Microstructure During Early NREM Sleep," Brain Topogr., vol. 28, no. 4, pp. 619-635, 2015.

[20] I. M. Comsa, T. A. Bekinschtein, and S. Chennu, "Transient Topographical Dynamics of the Electroencephalogram Predict Brain Connectivity and Behavioural Responsiveness During Drowsiness," Brain Topogr., vol. 32, no. 2, pp. 315-331, 2019.

[21] H. Katayama et al., "Classes of multichannel EEG microstates in light and deep hypnotic conditions," Brain Topogr., vol. 20, no. 1, pp. 7-14, 2007.

[22] J. Breshears et al., "Stable and dynamic cortical electrophysiology of induction and emergence with propofol anesthesia," Proc. Natl. Acad. Sci., vol. 107, no. 49, pp. 21170-21175, 2010.

[23] R. Baker et al., "Altered Activity in the Central Medial Thalamus Precedes Changes in the Neocortex during Transitions into Both Sleep and Propofol Anesthesia," J. Neurosci., vol. 34, no. 40, pp. 13326-13335, 2014

[24] S. Vijayan, S. Ching, P. L. Purdon, E. N. Brown, and N. J. Kopell, "Thalamocortical Mechanisms for the Anteriorization of Alpha Rhythms during Propofol-Induced Unconsciousness," J. Neurosci., vol. 33, no. 27, pp. 11070-11075, 2013.

[25] R. D. Pascual-marqui, C. M. Michel, and D. Lehmann, "Segmentation of Brain Electrical Activity into Microstates: Model Estimation and Validation," IEEE Trans. Biomed. Eng., vol. 42, no. 7, pp. 658-665, 1995.

[26] D. P. Mandic, N. Ur Rehman, Z. Wu, and N. E. Huang, "Empirical mode decomposition-based time-frequency analysis of multivariate signals: The power of adaptive data analysis," IEEE Signal Process. Mag., vol. 30, no. 6, pp. 74-86, 2013.

[27] P. Milz, P. Achermann, K. Kochi, and P. L. Faber, "The EEG microstate topography is predominantly determined by intracortical sources in the alpha band," Neuroimage, vol. 162, pp. 353-361, 2017.

[28] N. Huang et al., "The empirical mode decomposition and the Hilbert spectrum for nonlinear and non-stationary time series analysis," Proc. R. Soc. A, vol. 454, pp. 679-699, 1998.

[29] W. Shi et al., "Non-Canonical Microstate Becomes Salient in High Density EEG During Propofol-Induced Altered States of Consciousness," Int. J. Neural Syst., vol. 30, no. 2, p. 2050005, 2020.

[30] N. Huang and Z. Wu, "A Review on Hilbert-Hung Transform Method and Its Applications to Geophysical Studies," Rev. Geophys., vol. 46, no. 2, 2008.

[31] A. Delorme and S. Makeig, "EEGLAB : an open source toolbox for analysis of single-trial EEG dynamics including independent component analysis," J. Neurosci. Methods, vol. 134, pp. 9-21, 2004. M. M. Murray, D. Brunet, and C. M. Michel, "Topographic ERP analyses: A step-by-step tutorial review," Brain Topogr., vol. 20, no. 4, pp. 249-264, 2008.

[33] A. Custo, D. Van De Ville, W. M. Wells, M. I. Tomescu, D. Brunet, and C. M. Michel, "Electroencephalographic Resting-State Networks: Source Localization of Microstates," Brain Connect., vol. 7, no. 10, pp. 671-682, 2017.

[34] C. M. Michel and T. Koenig, "EEG microstates as a tool for studying the temporal dynamics of whole-brain neuronal networks: A review," Neuroimage, vol. 180, pp. 577-593, 2018.

[35] P. Milz, P. L. Faber, D. Lehmann, T. Koenig, K. Kochi, and R. D. Pascual-marqui, "The functional significance of EEG microstates Associations with modalities of thinking," Neuroimage, vol. 125, pp. 643-656, 2016.

[36] N. ur Rehman, Y. Xia, and D. P. Mandic, "Application of multivariate empirical mode decomposition for seizure detection in EEG signals," in 2010 Annu. Int. Conf. IEEE Eng. Med. Biol., 2010, pp. $1650-1653$.

[37] G. Rilling, P. Flandrin, P. Gonçalves, and J. M. Lilly, "Bivariate Empirical Mode Decomposition," IEEE Signal Process. Lett., vol. 14, no. 12, pp. 936-939, 2007.
[38] N. Rehman and D. P. Mandic, "Multivariate empirical mode decomposition," Proc. R. Soc. A Math. Phys. Eng. Sci., vol. 466, no. 2117, pp. 1291-1302, 2010.

[39] Y. Morimoto, S. Hagihira, Y. Koizumi, K. Ishida, M. Matsumoto, and T. Sakabe, "The Relationship Between Bispectral Index and Electroencephalographic Parameters During Isoflurane Anesthesia," Anesth. Analg., vol. 98, no. 5, pp. 1336-1340, 2004.

[40] S. Hagihira, M. Takashina, T. Mori, T. Mashimo, and I. Yoshiya, "Practical issues in bispectral analysis of electroencephalographic signals," Anesth. Analg., vol. 93, no. 4, pp. 966-970, 2001.

[41] J. Britz, D. Van De Ville, and C. M. Michel, "BOLD correlates of EEG topography reveal rapid resting-state network dynamics," Neuroimage, vol. 52, no. 4, pp. 1162-1170, 2010.

[42] M. Murphy et al., "Propofol anesthesia and sleep: a high-density EEG study.," Sleep, vol. 34, no. 3, pp. 283-91, 2011.

[43] P. L. Purdon, A. Sampson, K. J. Pavone, and E. N. Brown, "Clinical Electroencephalography for Anesthesiologists Part I: Background and Basic Signatures," Anesthesiology, vol. 123, no. 4, pp. 937-960, 2015.

[44] L. D. Lewis et al., "Rapid fragmentation of neuronal networks at the onset of propofol-induced unconsciousness," Proc. Natl. Acad. Sci., vol. 109, no. 49, pp. 19891-19892, 2012.

[45] I. J. Rampil and R. S. Matteo, "Changes in EEG Spectral Edge Frequency Correlate with the Hemodynamic Response to Laryngoscopy and Intubation," Anesthesiology, vol. 67, no. 1, pp. 139-142, 1987.

[46] P. Boveroux, A. Vanhaudenhuyse, B. Sc, B. Sc, C. Phillips, and D. $\mathrm{Ph}$, "Breakdown of within- and between-network Resting State during Propofol-induced Loss of Consciousness," Anesthesiology, vol. 113 , no. 5 , pp. 1038-53, 2010 .

[47] X. Liu, K. Lauer, B. Douglas Ward, S.-J. Li, and A. Hudetz, "Differential Effects of Deep Sedation with Propofol on the Specific and Nonspecific Thalamocortical Systems A Functional Magnetic Resonance Imaging Study," Anesthesiology, vol. 118, no. 1, pp. 59-69, 2013.

[48] R. Ní Mhuircheartaigh, D. Rosenorn-Lanng, R. Wise, S. Jbabdi, R. Rogers, and I. Tracey, "Cortical and Subcortical Connectivity Changes during Decreasing Levels of Consciousness in Humans: A Functional Magnetic Resonance Imaging Study using Propofol," $J$. Neurosci., vol. 30, pp. 9095-9102, 2010.

[49] M. Boly et al., "Connectivity Changes Underlying Spectral EEG Changes during Propofol-Induced Loss of Consciousness," $J$. Neurosci., vol. 32, no. 20, pp. 7082-7090, 2012.

[50] B. A. Seitzman, M. Abell, S. C. Bartley, M. A. Erickson, A. R. Bolbecker, and W. P. Hetrick, "Cognitive manipulation of brain electric microstates," Neuroimage, vol. 146, pp. 533-543, 2017.

[51] G. Plourde et al., "Cortical Processing of Complex Auditory Stimuli during Alterations of Consciousness with the General Anesthetic," Anesthesiology, vol. 104, no. 3, pp. 448-457, 2006.

[52] L. Xun, A. Horch, and H. Su, "Fast Multivariate Empirical Mode Decomposition," IEEE Access, vol. 6, pp. 65521-65538, 2018. 